Pacific Journal of Mathematic

av rans Esumu ance 


\section{ON PRIME GAMMA RINGS}

\section{SHOJI KYUNO}

The notion of a $\Gamma$-ring was introduced by $N$. Nobusawa. The class of $\Gamma$-rings contains not only all rings but also Hestenes ternary rings. Recently, W. E. Barnes, J. Luh, $W$. E. Coppage and the author studied the structure of $\Gamma$-rings and obtained various generalizations analogous of corresponding parts in ring theory. The object of this paper is to study the properties of prime $\Gamma$-rings. Main results are the following theorems: (1) A $\Gamma$-ring $M$ is a subdirect sum of prime $\Gamma$-rings if and only if $\mathscr{P}(M)=0$, where $\mathscr{P}(M)$ denotes the prime radical of M. (2) For the matrix $\Gamma_{n, m}$-ring $M_{m, n}$ we have $\mathscr{P}\left(M_{m, n}\right)=$ $(\mathscr{P}(M))_{m, n}$, where $M$ is a ring such that $x \in M \Gamma x \Gamma M$ for every $x \in M$.

2. Preliminaries. Let $M$ and $\Gamma$ be two abelian groups. If for all $x, y, z \in M$ and all $\alpha, \beta \in \Gamma$, the conditions (1) $x \alpha y \in M$ (2) $(x+y) \alpha z=x \alpha z+y \alpha z, x(\alpha+\beta) z=x \alpha z+x \beta z, x \alpha(y+z)=x \alpha y+x \alpha z$, (3) $(x \alpha y) \beta z=x \alpha(y \beta z)$ are satisfied, then we call $M$ a $\Gamma$-ring.

If $A$ and $B$ are subsets of a $\Gamma$-ring $M$ and $\Theta \subseteq \Gamma$, we denote $A \Theta B$, the subset of $M$ consisting of all finite sums of the form $\Sigma a_{i} \gamma_{i} b_{i}$ where $a_{i} \in A, b_{i} \in B$ and $\gamma_{i} \in \Theta$. For singleton subsets we abbreviate this notation for example, $\{a\} \Theta B=a \Theta B$. A right ideal (left ideal) of a $\Gamma$-ring $M$ is an additive subgroup $I$ of $M$ such that $I \Gamma M \subseteq I$ $(M \Gamma I \subseteq I)$. If $I$ is both a right and a left ideal, then we say that $I$ is an ideal, or two-sided ideal of $M$. For each $a$ of a $\Gamma$-ring $M$, the smallest right ideal containing $a$ is called the principal right ideal generated by $a$ and is denoted by $|a\rangle$. Similarly we define $\langle a|$ and $\langle a\rangle$, the principal left and two-sided (respectively) ideals generated by $a$.

Let $I$ be an ideal of a $\Gamma$-ring $M$. If for each $a+I, b+I$ in the factor group $M / I$, and each $\gamma \in \Gamma$, we define $(a+I) \gamma(b+I)=a \gamma b+I$, then $M / I$ is a $\Gamma$-ring which we shall call the $\Gamma$-residue class ring of $M$ with respect to $I$.

If $M_{i}$ is a $\Gamma_{i}$-ring for $i=1,2$ then an ordered pair $(\theta, \phi)$ of mappings is called a homomorphism of $M_{1}$ onto $M_{2}$ if it satisfies the following properties: (1) $\theta$ is a group homomorphism from $M_{1}$ onto $M_{2}(2) \phi$ is a group isomorphism from $\Gamma_{1}$ onto $\Gamma_{2}$ (3) For every $x, y \in M_{1}, \gamma \in \Gamma_{1}$, $(x \gamma y) \theta=(x \theta)(\gamma \phi)(y \theta)$. The kernel of the homomorphism $(\theta, \phi)$ is defined to be $K=\{x \in M \mid x \theta=0\}$. Clearly $K$ is an ideal of $M$. If $\theta$ is a group isomorphism, that is, if $K=0$, then $(\theta, \phi)$ is called an isomorphism from the $\Gamma_{1}$-ring $M_{1}$ onto the $\Gamma_{2}$-ring $M_{2}$.

Let $I$ be an ideal of the $\Gamma$-ring $M$. Then the ordered pair $(\rho, \iota)$ of 
mappings, where $\rho: M \rightarrow M / I$ is defined by $x \rho=x+I$ and $\iota$ is the identity mapping of $\Gamma$, is a homomorphism called the natural homomorphism from $M$ onto $M / I$.

For all other notions relevant to $\Gamma$-rings we refer to [4].

\section{Semi-primeness.}

Definitions. An ideal $P$ of a $\Gamma$-ring $M$ is prime if for any ideals $A, B \subseteq M, A \Gamma B \subseteq P$ implies $A \subseteq P$ or $B \subseteq P$. A subset $S$ of $M$ is an $m$-system in $M$ if $S=\varnothing$ or if $a, b \in S$ implies $\langle a\rangle \Gamma\langle b\rangle \cap S \neq \varnothing$. The prime radical $\mathscr{P}(A)$ is the set of $x$ in $M$ such that every $m$-system containing $x$ meets $A$. The prime radical of the zero ideal in a $\Gamma$-ring $M$ is called the prime radical of the $\Gamma$-ring $M$ which we denote by $\mathscr{P}(M)$. An ideal $Q$ of $M$ is semiprime if, for any ideal $U, U \Gamma U \subseteq Q$ implies $U \subseteq Q$. A $\Gamma$-ring $M$ is semi-prime if the zero ideal is semiprime.

The following theorem characterizes semi-primeness for ideals in $\Gamma$-rings. The proof is a minor modification of the proof of the corresponding theorem in ring theory, and we omit it.

THEOREM 1. If $Q$ is an ideal in a $\Gamma$-ring $M$, all the following conditions are equivalent.

(1) $Q$ is a semi-prime ideal.

(2) If $a \in Q$ such that $a \Gamma M \Gamma a \subseteq Q$, then $a \in Q$. $a \in Q$.

(3) If $\langle a\rangle$ is a principal ideal in $M$ such that $\langle a\rangle \Gamma\langle a\rangle \subseteq Q$, then

(4) If $U$ is a right ideal in $M$ such that $U \Gamma U \subseteq Q$, then $U \subseteq Q$.

(5) If $V$ is a left ideal in $M$ such that $V \Gamma V \subseteq Q$, then $V \subseteq Q$.

COROllary 1. A $\Gamma$-ring $M$ is semi-prime if and only if $a \Gamma M \Gamma a=$ 0 implies $a=0$.

Definition. A subset $S$ of $M$ is strongly nilpotent if there exists a positive integer $n$ such that $(S \Gamma)^{n} S=(0)$.

It follows easily by induction that if $Q$ is a semi-prime ideal and $A$ is an ideal such that $(A \Gamma)^{n} A \subseteq Q$ for an arbitrary positive integer $n$, then $A \subseteq Q$. Hence, (0) is a semi-prime ideal if and only if $M$ contains no nonzero strongly nilpotent ideal. By Theorem 1 (4) and (5), we have also that $(0)$ is a semi-prime ideal if and only if $M$ contains no nonzero strongly nilpotent right (left ideal).

The author [3] showed the following result. 
THEOREM 2. An ideal $Q$ in a $\Gamma$-ring $M$ is a semi-prime ideal in $M$ if and only if $\mathscr{P}(Q)=Q$.

By Theorem 2, (0) is a semi-prime ideal if and only if $\mathscr{P}(M)=(0)$.

Thus we have the following theorem.

THEOREM 3. A $\Gamma$-ring $M$ has zero prime radical if and only if it contains no strongly nilpotent ideal (right ideal, left ideal).

4. Prime $\Gamma$-rings. In this section we shall be concerned with the concept introduced in the following definition.

Definition. A $\Gamma$-ring $M$ is said to be prime if the zero ideal is prime.

The following theorem is analogous to the corresponding theorem in ring theory, and we omit its proof.

THEOREM 4. If $M$ is a $\Gamma$-ring, the following conditions are equivalent:

(1) $M$ is a prime $\Gamma$-ring.

(2) If $a, b \in M$ and $a \Gamma M \Gamma b=(0)$, then $a=0$ or $b=0$.

(3) If $\langle a\rangle$ and $\langle b\rangle$ are principal ideals in $M$ such that $\langle a\rangle \Gamma\langle b\rangle=(0)$, then $a=0$ or $b=0$.

(4) If $A$ and $B$ are right ideals in $M$ such that $A \Gamma B=(0)$, then $A=(0)$ or $B=(0)$.

(5) If $A$ and $B$ are left ideals in $M$ such that $A \Gamma B=(0)$, then $A=(0)$ or $B=(0)$.

The importance of the concept of prime $\Gamma$-rings stems primarily from the following fact.

THEOREM 5. If $P$ is an ideal in the $\Gamma$-ring $M$, then the $\Gamma$-residue class ring $M / P$ is a prime $\Gamma$-ring if and only if $P$ is a prime ideal in $M$.

We prepare the following lemma which is fairly easy to prove, and we omit the proof.

LemMa 1. Let $(\theta, \iota)$ be a homomorphism of $\Gamma$-ring $M$ onto the $\Gamma$-ring $N$, with kernel $K$. Then each of the following is true: in $N$.

(1) If $I$ is an ideal (right ideal) in $M$, then IO is an ideal (right ideal) 
(2) If $J$ is an ideal (right ideal) in $N$, then $J^{-1}$ is an ideal (right ideal) in $M$ which contains $K$.

(3) If $I$ is an ideal (right ideal) in $M$ which contains $K$, then $I=(I \theta) \theta^{-1}$.

(4) The mapping $I \rightarrow I \theta$ defines a one to one mapping of the set of ideals (right ideals) in $M$ which contain $K$ onto the set of all ideals (right ideals) in $N$.

Proof of Theorem 5. Let $M / P$ be prime and $A, B$ be ideals of $M$ such that $A \Gamma B \subseteq P$. Let $(\rho, \iota)$ be the natural homomorphism from $M$ onto $M / P$. Then by Lemma $1 A \theta$ and $B \theta$ are ideals of $M / P$ such that $A \theta \Gamma B \theta=(0)$. Since $M / P$ is prime, it follows that $A \theta=(0)$ or $B \theta=(0)$, that is, $A \subseteq P$ or $B \subseteq P$. Thus $P$ is a prime ideal in $M$.

Conversely, let $P$ be a prime ideal in $M$. Lemma 1 shows that each ideal in $M / P$ is of the form $A / P$, where $A$ is an ideal in $M$ which contains $P$. Thus we may assume that $A / P, B / P$ be ideals of $M / P$ such that $(A / P) \Gamma(B / P)=(0)$, which implies $A \Gamma B \subseteq P$. Then by the primeness of $P$ we have $A \subseteq P$ or $B \subseteq P$. Hence $A=P$ or $B=P$ and so $A / P=(0)$ or $B / P=(0)$. This completes the proof.

Barnes [1] has characterized $\mathscr{P}(M)$ as the intersection of all prime ideals of $M$.

The author [4] has shown the following lemma.

LEMMA 2. A $\Gamma$-ring $M$ is a subdirect sum of $\Gamma$-rings $S_{i}, i \in \mathfrak{U}$, if and only if for each $i \in \mathfrak{A}$ there exists in $M$ a two-sided ideal $K_{i}$ such that $M / K_{t} \cong S_{i}$, moreover $\bigcap_{i \in \mathfrak{U}} K_{i}=(0)$.

Thus, these facts and Theorem 5 yield the following theorem which is analogous to Theorem 4.3 in [4].

THEOREM 6. A $\Gamma$-ring $M$ is a subdirect sum of prime $\Gamma$-rings if and only if $\mathscr{P}(M)=(0)$.

Following Luh [2], we introduce the matrix ring $M_{m, n}$.

Let $G$ be an additive group. We shall denote by $G_{m, n}$ the additive group of all $m \times n$ matrices over the group $G$. For $1 \leqq i \leqq m, 1 \leqq j \leqq n$, and $a \in G$, let $a E_{i j}$ denote the matrix having $a$ at the $i$ th row and $j$ th column, and 0 elsewhere.

Let $M$ be a $\Gamma$-ring. Consider the group $M_{m, n}$ and $\Gamma_{n, m}$. For $\left(a_{i j}\right),\left(b_{i j}\right) \in M_{m, n}$ and $\left(\gamma_{i j}\right) \in \Gamma_{n, m}$, define $\left(a_{i j}\right)\left(\gamma_{i j}\right)\left(b_{i j}\right)=\left(c_{i j}\right)$, where $c_{i j}=$ $\sum_{k=1}^{m} \sum_{h=1}^{n} a_{i h} \gamma_{h k} b_{k j}$. Then $M_{m, n}$ forms a $\Gamma_{n, m}$-ring.

We now prove the next theorem which will indicate one way to construct new prime $\Gamma$-rings from given ones. 
THEOREM 7. If $M$ is a $\Gamma$-ring, the matrix ring $M_{m, n}$ is a prime $\Gamma_{n, m}$-ring if and only if $M$ is a prime $\Gamma$-ring.

Proof. Let us prove that if $M$ is not prime, then $M_{m, n}$ is not prime. If $M$ is not prime, there exist nonzero elements $a$ and $b$ of $M$ such that $a \Gamma M \Gamma b=0$. Then, we have, for example, $a E_{11} \Gamma_{n, m} M_{m, n} \Gamma_{n, m} b E_{11}=0$ with $a E_{11}$ and $b E_{11}$ nonzero elements of $\boldsymbol{M}_{m, n}$. Hence, $\boldsymbol{M}_{m, n}$ is not prime. Conversely, suppose that $\boldsymbol{M}_{m, n}$ is not prime, and hence that there exist nonzero matrices $\Sigma_{i, j} a_{i j} E_{i j}$ and $\Sigma_{i, j} b_{i j} E_{i j}$ such that $\left(\sum_{i, j} a_{i j} E_{i j}\right) \Gamma_{n, m} M_{m, n} \Gamma_{n, m}\left(\Sigma_{i, j} b_{i j} E_{i j}\right)=0$. Let $p, q, r$ and $s$ be fixed positive integers such that $a_{p, q} \neq 0$ and $b_{r s} \neq 0$. As a special case of the preceding equation, we find that for each $x \in M$, each $\gamma, \eta \in \Gamma$,

$$
\left(\Sigma a_{i j} E_{i j}\right)\left(\gamma E_{q p}\right)\left(x E_{p s}\right)\left(\eta E_{s r}\right)\left(\Sigma b_{i j} E_{i j}\right)=\Sigma a_{i q} \gamma x \eta b_{r j} E_{i j}=0 .
$$

In particular, the $(p, s)$ element must be zero, that is, $a_{p q} \gamma x \eta b_{r s}=$ 0 . Since this is true for every $x$ in $M$ and every $\gamma, \eta$ in $\Gamma$, we have $a_{p q} \Gamma M \Gamma b_{r s}=0$, and $M$ is not prime. This completes the proof.

Luh [2] has obtained the following lemma.

LeMMA 3. Let $M$ be a $\Gamma$-ring such that $x \in M \Gamma x \Gamma M$ for every $x \in M$. Then the ideals of the $\Gamma_{n, m}$-ring $M_{m, n}$ are the form $U_{m, n}$, where $U$ is an ideal of $M$.

We prepare the following lemma.

LEMMA 4. If $I$ is an ideal in the $\Gamma$-ring $M$, then the matrix $\Gamma_{n, m}$-ring $(M / I)_{m, n}$ is isomorphic to the $\Gamma_{n, m}-$ ring $M_{m, n} / I_{m, n}$.

Proof. Let $\theta$ be a mapping of the $\Gamma_{n, m}$-ring $(M / I)_{m, n}$ to the $\Gamma_{n, m}$-ring $M_{m, n} / I_{m, n}$ such that $\left(x_{i j}+I\right) \theta=\left(x_{i j}\right)+I_{m, n}$. Clearly, $\theta$ is a group isomorphism from $(M / I)_{m, n}$ onto $M_{m, n} / I_{m, n}$. Let $\iota$ be an identity mapping from $\Gamma_{n, m}$ onto $\Gamma_{n, m}$. By the definition of multiplications of the $\Gamma$-residue class ring, we have that

$$
\begin{aligned}
{\left[\left(x_{i j}+I\right)\left(\gamma_{i j}\right)\left(y_{i j}+I\right)\right] \theta } & =\left(z_{i j}+I\right) \theta, \text { where }\left(z_{i j}\right)=\left(x_{i j}\right)\left(\gamma_{i j}\right)\left(y_{i j}\right) \\
& =\left(x_{i j}\right)\left(\gamma_{i j}\right)\left(y_{i j}\right)+I_{m, n} \\
& =\left[\left(x_{i j}\right)+I_{m, n}\right]\left(\gamma_{i j}\right)\left[\left(y_{i j}\right)+I_{m, n}\right] \\
& =\left(x_{i j}+I\right) \theta\left(\gamma_{i j}\right) \iota\left(y_{i j}+I\right) \theta .
\end{aligned}
$$

This shows that $(\theta, \iota)$ is an isomorphism of $(M / I)_{m, n}$ onto $M_{m, n} / I_{m, n}$.

We now prove the following result. 
THEOREM 8. Let $M$ be a $\Gamma$-ring such that $x \in M \Gamma x \Gamma M$ for every $x \in M$. If $\mathscr{P}(M)$ is the prime radical of the $\Gamma$-ring $M$, then $\mathscr{P}\left(M_{m, n}\right)=$ $(\mathscr{P}(M))_{m, n}$.

Proof. From Lemma 3 it follows easily that $I \rightarrow I_{m, n}$ ( $I$ an ideal in $M)$ is a one to one mapping of the set of all ideals in $M$ onto the set of all ideals in $M_{m, n}$. Moreover, by Lemma $4,(M / I)_{m, n} \cong M_{m, n} / I_{m, n}$. Hence, by Theorem 7, $M_{m, n} / I_{m, n}$ is a prime $\Gamma_{n, m}$-ring if and only if $M / I$ is a prime $\Gamma$-ring. From Theorem 5 it follows that $I_{m, n}$ is a prime ideal of $M_{m, n}$ if and only if $I$ is a prime ideal of $M$. Thus, if $\left\{P_{i} \mid i \in \mathfrak{U}\right\}$ is the set of all prime ideals in $M$, we have

$$
\mathscr{P}\left(M_{m, n}\right)=\bigcap_{i \in \mathscr{U}}\left(P_{i}\right)_{m, n}=\left(\bigcap_{i \in \mathscr{A}} P_{i}\right)_{m, n}=(\mathscr{P}(M))_{m, n} .
$$

Remarks. A $\Gamma$-ring $M$ is said to be simple if (1) $M \Gamma M \neq 0$ and (2) $M$ has no ideals other than 0 and $M$ itself. If $M$ is simple, $M \Gamma x \Gamma M=$ $M$ for each nonzero element $x$ in $M$. Hence $x \in M \Gamma x \Gamma M$. Thus, for a simple $\Gamma$-ring $M, \mathscr{P}\left(M_{m, n}\right)=(\mathscr{P}(M))_{m, n}=0$.

If there exists an element $\epsilon$ in $M$ and an element $\delta$ in $\Gamma$ such that $x \partial \epsilon=\epsilon \partial x=x$ for every element $x \in M, \epsilon$ is called an unity of $M$. If $M$ has an unity, for every $x$ in $M x \in M \Gamma x \Gamma M$, and then $\mathscr{P}\left(M_{m, n}\right)=$ $(\mathscr{P}(M))_{m, n}$.

\section{REFERENCES}

1. W. E. Barnes, On the Г-rings of Nobusawa, Pacific J. Math., 18 (1966), 411-422.

2. J. Luh, On the theory of simple $\Gamma$-rings, Michigan Math. J., 16 (1969), 65-75.

3. S. Kyuno, On the radicals of $\Gamma$-rings, Osaka J. Math., 12 (1975), 639-645.

4. S. Kyuno, On the semi-simple gamma rings, Tohoku Math. J., 29 (1977), 217-225.

5. N. Nobusawa, On a generalization of the ring theory, Osaka J. Math., 1 (1964), 81-89.

Received March 9, 1977.

TOHOKU GAKUIN UNIVERSITY

AsahigaOKa TAgajo-City, 985

JAPAN 


\section{PACIFIC JOURNAL OF MATHEMATICS EDITORS}

ICHARD ARENS (Managing Editor)

niversity of California

os Angeles, CA 90024

\section{. A. BEAUmont}

niversity of Washington

sattle, WA 98105

. C. MOORE

niversity of California

erkeley, CA 94720
J. DUGUNDJI

Department of Mathematics

University of Southern California

Los Angeles, CA 90007

R. FINN AND J. MiLgRAM

Stanford University

Stanford, CA 94305

\section{ASSOCIATE EDITORS}
F. BECKENBACH
B. H. NEUMANN
F. WOLF
K. YosHidA

\section{SUPPORTING INSTITUTIONS}

NIVERSITY OF BRITISH COLUMBIA

UNIVERSITY OF SOUTHERN CALIFORNIA

ALIFORNIA INSTITUTE OF TECHNOLOGY STANFORD UNIVERSITY

NIVERSITY OF CALIFORNIA

ONTANA STATE UNIVERSITY

UNIVERSITY OF HAWAII

NIVERSITY OF NEVADA

UNIVERSITY OF TOKYO

EW MEXICO STATE UNIVERSITY

UNIVERSITY OF UTAH

REGON STATE UNIVERSITY

NIVERSITY OF OREGON

WASHINGTON STATE UNIVERSITY

UNIVERSITY OF WASHINGTON

SAKA UNIVERSITY

AMERICAN MATHEMATICAL SOCIETY

The Supporting Institutions listed above contribute to the cost of publication of this Journal, but they a t owners or publishers and have no responsibility for its contents or policies.

Mathematical papers intended for publication in the Pacific Journal of Mathematics should be in typ Irm or offset-reproduced (not dittoed), double spaced with large margins. Underline Greek letters in re ierman in green, and script in blue. The first paragraph or two must be capable of being used separately as 'nopsis of the entire paper. Items of the bibliography should not be cited there unless absolutely necessary, hich case they must be identified by author and Journal, rather than by item number. Manuscripts, uplicate, may be sent to any one of the four editors. Please classify according to the scheme of Math. Revieu Idex to Vol. 39. All other communications should be addressed to the managing editor, or Elaine Bart Iniversity of California, Los Angeles, California, 90024.

100 reprints are provided free for each article, only if page charges have been substantially pai dditional copies may be obtained at cost in multiples of 50 .

The Pacific Journal of Mathematics is issued monthly as of January 1966. Regular subscription rate: \$72. year (6 Vols., 12 issues). Special rate: $\$ 36.00$ a year to individual members of supporting institutions.

Subscriptions, orders for numbers issued in the last three calendar years, and changes of address should ent to Pacific Journal of Mathematics, 103 Highland Boulevard, Berkeley, California, 94708.

UBLISHED BY PACIFIC JOURNAL OF MATHEMATICS, A NON-PROFIT CORPORATION

Printed at Jerusalem Academic Press, POB 2390, Jerusalem, Israel.

Copyright (C) 1978 Pacific Journal of Mathematics

All Rights Reserved 


\section{Pacific Journal of Mathematics}

\section{Vol. 75, No. $1 \quad$ September, 1978}

Mieczyslaw Altman, General solvability theorems

Denise Amar and Eric Amar, Sur les suites d'interpolation en plusieurs variables ..........................................

Herbert Stanley Bear, Jr. and Gerald Norman Hile, Algebras which satisfy a second order linear partial differential equation ..................

Marilyn Breen, Sets in $R^{d}$ having $(d-2)$-dimensional kernels ............

Gavin Brown and William Moran, Analytic discs in the maximal ideal space

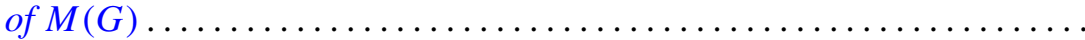

Ronald P. Brown, Quadratic forms with prescribed Stiefel-Whitney

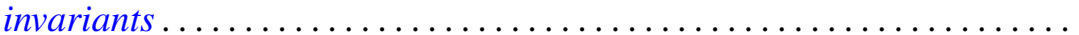

Gulbank D. Chakerian and H. Groemer, On coverings of Euclidean space by convex sets

S. Feigelstock and Z. Schlussel, Principal ideal and Noetherian groups.....

Ralph S. Freese and James Bryant Nation, Projective lattices ............

Harry Gingold, Uniqueness of linear boundary value problems for

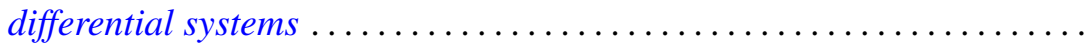

John R. Hedstrom and Evan Green Houston, Jr., Pseudo-valuation domains. . .

William Josephson, Coallocation between lattices with applications to measure extensions

M. Koskela, A characterization of non-negative matrix operators on $l^{p}$ to $l^{q}$ with $\infty>p \geq q>1$

Kurt Kreith and Charles Andrew Swanson, Conjugate points for nonlinear differential equations...........................

Shoji Kyuno, On prime gamma rings ........................ 185

Alois Andreas Lechicki, On bounded and subcontinuous multifunctions ..

Roberto Longo, A simple proof of the existence of modular automorphisms in approximately finite-dimensional von Neumann algebras ...

Kenneth Millett, Obstructions to pseudoisotopy implying isotopy for

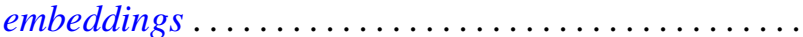

William F. Moss and John Piepenbrink, Positive solutions of elliptic equations. .

Mitsuru Nakai and Leo Sario, Duffin's function and Hadamard's

conjecture

Mohan S. Putcha, Word equations in some geometric semigroups ...

Walter Rudin, Peak-interpolation sets of class $C^{1} \ldots \ldots \ldots$

Elias Saab, On the Radon-Nikodým property in a class of locally convex

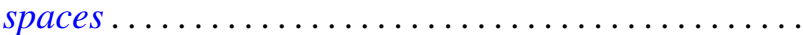

